\title{
Note sur les phénomènes périodiques dans les conduites forcées à caractéristiques multiples
}

\author{
par Charles JAEGER, Ingénieur, Docteur ès-Sciences techniques
}

\section{INTRODUCTION}

Un nombre considérable de travaux ont paru, au cours des dernières années, sur le problème du coup de bélier. Des raisons sérieuses les justifient. Les mêmes difficultés techniques, rencontrées en divers pays, ont suscité, entre spécialistes, une sorte d'émulation dans la recherche; certains problèmes ont mème été traités et résolus, presque simultanément el de façon analogue, par des auteurs fravaillant indépendamment les uns des autres. Nous constatons avec plaisir que leurs résultats se recoupent.

Il est, en effet, un point sur lequel nous insisterons : c'est l'unité et la concordance certaine des diverses méthodes de calcul employées. Récemment, deux auteurs connus (1) les ont mises en doute. Nous avons eu l'occasion, en leur répondant (2), de démontrer, au contraire, l'unité de la méthode analytique, mise au point par les travaux d'Allievi, de Sparre, Camichel et les nottres et de rappeler, en outre, la belle démonstration donnée par Bergeron, prouvant la parfaite concordance des méthodes graphiques BergeronSchnyder et des méthodes analytiques (3).

La théorie du coup de bélier peut, en effet, être exposée en employant soit les méthodes graphiques, dont le germe est contenu idans l'exposé de Lœwy (4), soit au moyen d'une étude analytique, 'dont les principes avaient été posés par Allievi et de Sparre. Certains auteurs n'ont foi qu'en la méthode analytique (5), d'autres, au contraire, préconisent le seul emploi des méthodes graphiques (6). Il y a là, de part et d'autre, quelque excès. Les deux méthodes nous paraissent également puissantes et souples ; les deux sont, de plus, également indispensables.

Voici, entre nombre, quelques preuves justifiant notre point do vue : Dans un récent article (6), Bergeron étudiait, au moyen de sa méthode graphique, "la fermeture napide d'un orifice sur une conduite munie d'une dérivation ". Or, dans un article antérieur de quelques mois à celui de Bergeron (7), no'us avions résolu le même exemple particulier, à titre d'application de la théorie analytique complète des conduites jumelées. Dans le même article de Bergeron, nous trouvons quelques cas de résonance de conduites simples

(1) Calane ex Gaden. - Considénations sur, le colp de bélier, Bulletin technique de la Suisse Romande, 14 sept. et 23 nor. 1935 , 29 février 1936 .

(2) Charles JAtger. - Quelques remarques en marge de la thério du coup de bẻlier, Bulletin technique de la Suisse Romande, 9 mai 1936.

(3) Bengenon. - Revue générale de l'Hydrautique, No 1, janvicr-février 1935, pages 21 et 22 , et No 2 , mars/avril 1935.

(4) Lowwy. - Druckschwankungen in Druckrohrleitungen, Vienne. Springer 1928.

(5) Alievr. - Arresto di una colonna liquikla in moto aseendente, L'Elettrotecnica, 25 octobre 1934, (voir : Osservazioni preliminari).

(6) Bergeron. - Etude des coups de bélier dans les conduites ; nouve] exposé de la méthode graphique. La technique moderne, Nos 2 et 3 (janvier-fóvrier 1936) (voir les "conclusions " de Bergeron).

(7) Charles JAEcisn. - Note sur lo coup de bélier dans les conduites jumelées on parallèles, Revec générale de l'Hydranlique $\mathrm{N}^{\circ} 3$, maijuin 1935 . également étudiés par voie graphique. Qu'on nous permette de rappeler, sur ce mème sujet, les fort belles recherches analytiques de Camichel, Eydoux et Gariel (9), bien antérieures aux travaux de Bergeron; ainsi que nos trois notes, que M. Fabry a eu l'obligeance de lire à l'Académie des Sciences au cours du printemps 1936 ; notes où nous abordions ces mêmes problèmes sous leur aspect le plus général (8).

Il nous semble donc vain, sous prétexle de faire valoir l'une des méthodes, graphique ou analytique, de mésestimer l'autre. Ce qui importe, c'est le progrès de nos connaissances communes dans le domaine qui fait l'objet de nos études. Nous pensons donc qu'il y a quelque utilité à développer les notes un peu sommaires que nous avons publiées, dans les Comples Rendus de l'Académie des Sciences, sur les phénomènes de résonance et les phénomènes périodiques dans les conduites à caractéristiques multiples (8).

Après les travaux d'Allievi, de Camichel-Eydoux-Gariel et ceux du Comte de Sparre, il paraîtra bien inutile de définir les résonances dans les conduites forcées. Précisons, par contre, que nous entendons par phénomènes périodiques les oscillations de charge qui se produisent. à titre de nhénomène secondaire, à la suite d'une mancuvre primaire d'ouverture ou de fermeture. Les phénomènes périodiques sont donc extrêmement fréquents. En outre, il y a une étroite parenté entre la résonance du fondamental dans une conduite quelconque et les mouvements périodiques. Il est donc tout à fait légitime de leur consacrer un même et seul chapitre. Si nous nous contentons d'aborder ici les seuls phénomènes périodiques, c'est afin de nes point allonger notre étude. Le lecteur pourra cependant se souvenir que notre travail n'est qu'une introduction à l'étude des résonances, ainsi qu'on peut le voir dans la seconde et troisième notes présentées à l'Académie.

\section{I. - PHENOMENES PERIODIQUES : \\ Galgul de la période apparente.}

Les expériences classiques de Camichel, Eydoux et $\mathrm{Ga}^{-}$ ricl (9) ont mis en relief les faits suivants :

Lorsqu'on étudie une conduite à caractéristique variable (par cx. à diamètre variable), au moyen dc la "méthode des dépressions brusques n de Camichel (10) -- méthode qui con-

(8) Charles Jaeger. - Theorie du coup de bélier dans les conduites forcées à caractéristiques multiples : Phénomènes périodiques ; résonance du fondamental et des harmoniques ; répartition des surcharges de résonanco lc long d"une conduite quelconque. Académie des Sciences, séancest des 27 février, 16 mars ct 4 mai 1936. C. R. tome 202.

(9) Camichel-Eydoux-Garmel. - "Etude théorique et expérimentale des coups de bélier " Dunod 1919.

(10) Camranes. - La Lumière Electrique, 9 et 16 sept. et 7 oct. 1916. ainsi que C. R. Tomo 161, pages 343 ct 412 , Tome 163 pages 150,224 et 438 . 
siste à provoquer au bas de ta conduite une brusque ouverture des vannes, suivie immédiatement d'une fermeture non moins brusque - on enregistre une période d'oscillation de la conduite, qui est la somme des périodes propres de chaque secteur de section constante de la conduite. Nous dirons, à la suite de Camichel, que celte période est la période d'oscillation théorique de la conduite.

$\mathrm{Si}$, au contraire, nous envisageons un mouvement, tel que le retour périodique du maximum de pression à la suite d'une fermeture lente de l'obturateur, on constate l'existence d'une période différente de la précédente, - en général, plus courte - dite période apparente de la conduite.

Etudiant, par voie expérimentale, les phénomènes de résonance dans les conduites à caractéristique variable, Camichel a vérifié que les harmoniques impairs des conduites forcées ont, comme période, une fraction entìre de la période d'oscillation théorique de la conduite, alors que la période du fondamental d'une conduite à caractéristique variable - fondamental qu'il est toujours possible de provoquer dans une pareille conduite - est, au contraire, égale à la période d'oscillation apparente.

Camichel et de Sparre (11) avaient estimé la période apparente d'une oscillation périodique d'une conduite à caractéristique variable, en calculant un nombre de maxima et de minima successifs suffisant pour qu'on puisse en déduire, avec une approximation satisfaisante, la période cherchée. Plusieuns mesures expérimentales, effectuées par Boucher (12), Neeser (13) et Camichel (14), ont vérifié les calculs de de Sparre et de Camichel. Ce procédé est évidemment un peu lent. Nous allons rechercher une méthode de calcul directe que nous pourrons généraliser et qui fait mieux ressortir le mécanisme du phénomène.

La période d'oscillation apparente, commune à tous les mouvements périodiques intéressant la longueur totale de la conduite, semble être une caractéristique de cette dernière, qu'il importe de connaître.

Il est parfaitement légitime de supposer - comme hypothèse de départ - que la courbe des surcharges à l'obturarateur est de forme sinusoïdale. L'observation d'un grand nombre de diagrammes de surcharges, pris au droit de l'obturateur de conduites à caractéristiques multiples, montre que, même dans les cas les plus complexes, les courbes primitivement d'allure compliquée tendent à devenir sinusoïdales. La courbe sinusoïdale est donc - c'est un fait d'observation - une solution possible du problème. Foch a même pu démontrer que, dans le cas de petites oscillations dans une conduite munie d'un réservoir d'air, la forme sinusoïdale est la seule possible (15). On pourrait, certes, rechercher quelles sont les formes de courbes susceptibles d'engendrer un mouvement périodique stable dans des systèmes complexes. Mais, cette recherche sorí du programme que nous nous sommes tracé et qui tend à rechercher les lois élémentaires des phénomènes périodiques et, plus particulièrement, la durée de la période apparente.

Rappelons les équations fondamentales du problème du coup de bélier :

(11) Lettre de de Sparre citée par Camaned, Ernoux, Garied.

(12) Conduite de Fully, Camraher, Eyooux, Gariel, p. $210-211$.

(13) Conduite de l'Akersand, Camiches, Eydoux, Garies, p. 211-212.

(14) Conduite de l'l. E. Toulouse, Camiches, Ermovx, Ganter, p. 219.

(15) Focr. - - Contribution à l'étude des cotups de bélier dans les con. duites munies d'un réservoir d'air \%. Toulouse, Ed. Privat 1920.

$$
\begin{gathered}
y=y_{0}+\mathrm{F}(t)+f(t) \\
\mathrm{V}=v_{0}-\frac{g}{a}[\mathrm{~F}(l)-f(t)],
\end{gathered}
$$

qui sont absolument générales, $\mathrm{F}$ el $f$ étant des fonctions d'intégration quelconques.

Posons donc, à l'obturateur :

$$
\begin{aligned}
& \text { (3) } \quad y-y_{0}=A \sin m t, \\
& \text { où } m=\frac{2 \pi}{\Theta}, \Theta \text { étant la période apparente cherchée. }
\end{aligned}
$$

Supposons que, l'obturateur étant fermé à partir du temps $t=o$, on ait constamment $v=0$, condition qui est effectivement réalisée au cours des mouvements périodiques que nous envisageons. On en tire :

$$
\begin{gathered}
v=-\frac{g}{a}[\mathrm{~F}(t)-f(t)]=0 ; \text { d'où : } \\
f(t)=\mathrm{F}(t)=\frac{\mathrm{A}}{2} \text { sin. } m t ;
\end{gathered}
$$

ce qui donne bien : $y-y_{0}=2 \mathrm{~F}(t)=\mathrm{A} \sin . m t$.

1) Cas d'une discontinuité de section (Voir fig. 1)

Au point de discontinuité $A$, on doit avoir continuité de la pression, d'où :

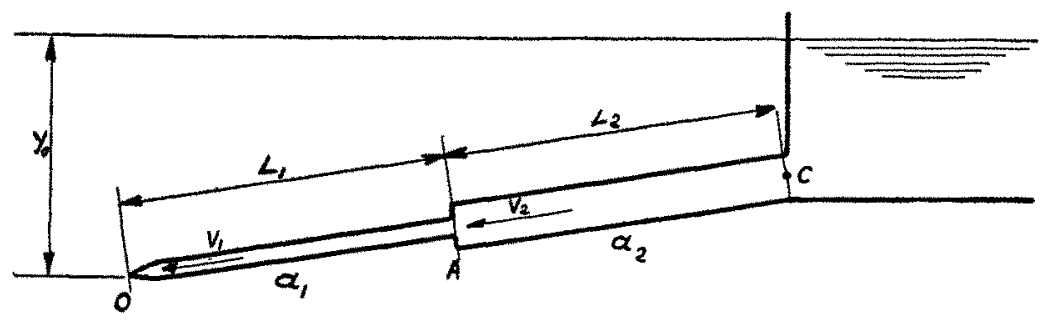

Fig. 1

$y_{1}=y_{2}$ et

$y_{1}-y_{10}=y_{2}-y_{20}$

les indices " 1 " et " 2 " se rapportant aux conduites " 1 " et $~ " 2$ ", le second indice " $o$ " se rapportant au régime normal.

La continuité des masses est exprimée par :

(6)

$$
\mathrm{S}_{1} v_{1}=\mathrm{S}_{2} v_{2}
$$

$S_{1}$ et $S_{2}$ représentant les sections des deux tronçons aval et amont de la conduite.

Nous écrivons, en appliquant l'équation fondamentale (1) au point de bifurcation $\mathrm{A}$, pour la conduite aval :

$$
\begin{gathered}
y_{1}-\gamma_{10}=\mathrm{F}\left(t--\frac{L_{1}}{a_{1}}\right)+f\left(t+\frac{L_{1}}{a_{1}}\right) \\
=\mathrm{F}\left(t-\frac{L_{1}}{a_{1}}\right)+\mathrm{F}\left(t+\frac{L_{1}}{a_{11}}\right)
\end{gathered}
$$

en tenant compte du retard du passage en $\mathrm{A}$, par rapport à $o$, de l'onde $F$ et de l'avance de l'onde $f$.

Pour la conduite amont, on a, par contre :

$$
y_{2}-y_{20}=\mathrm{G}(t)+\mathrm{g}(t)=G(t)-\mathrm{G}\left(t-\frac{2 \mathrm{~L}_{2}}{a_{2}}\right)
$$

en admettant qu'il y a réflexion totale à l'extrémité amont de la conduite et en désignant par $\mathrm{G}(t)$ et $g(t)$ deux fonctions d'intégration du même type que $\mathrm{F}(t)$ et $f(t)$.

Egalons les deux valeurs de $y_{1}$ et $y_{2}$ au point de discontinuité : 
(7) $\mathrm{F}\left(t-\frac{\mathrm{L} \cdot}{a_{1}}\right)+\mathrm{F}\left(t+\frac{\mathrm{L}_{1}}{a_{1}}\right)=\mathrm{G}(t)-\mathrm{G}\left(t-\frac{2 \mathrm{~L}_{2}}{\mathrm{a}_{2}}\right)$ posons :

$$
\mathrm{G}(t)=-\frac{B}{2} \cos (m t+\varphi),
$$

$B$ et $\varphi$ étant des constantes à déterminer.

Les équations (7) et (8) nous donnent :

$$
\begin{aligned}
& \frac{\mathrm{A}}{2} \sin m\left(l-\frac{L_{1}}{\mathrm{a}_{1}}\right)+\frac{A}{2} \sin m\left(t+\frac{L_{1}}{a_{2}}\right)= \\
& =-\frac{\mathrm{B}}{2} \cos (m t+\varphi)+\frac{\mathrm{B}}{2} \cos \left[m\left(t-\frac{2 L_{2}}{a_{2}}\right)+\varphi\right]
\end{aligned}
$$

(9) $\mathrm{A} \cos m \frac{\mathrm{L}_{1}}{a_{1}} \sin m t=\mathrm{B} \sin m \frac{\mathrm{L}_{2}}{a_{2}} \sin \left[m t+\varphi-\frac{m \mathrm{~L}_{2}}{a_{12}}\right]$

Pour que cette égalité soit satisfaite quel que soit $t$, il faut que :

et :

$$
\mathrm{A} \cos m \frac{\mathrm{L}_{1}}{e_{1}}=\mathrm{B} \sin m \frac{\mathrm{L}_{2}}{a_{2}}
$$

ou : $\left(\begin{array}{lll}10 & a\end{array}\right)$

$$
m t=m t+\varphi-m \frac{\mathrm{L}_{2}}{a_{2}}
$$

$$
\varphi=m \frac{L_{2}}{a_{2}}
$$

Ecrivons que la vitesse $v_{\imath}$ est donnée par :

$$
v_{1}=v_{1}-\frac{9}{a_{1}}\left[\mathrm{~F}\left(t-\frac{\mathrm{L}_{1}}{a_{1}}\right)-\mathrm{F}\left(t+\frac{\mathrm{L}_{1}}{a_{1}}\right)\right]
$$

$=v_{1}-\frac{9}{a_{1}}\left[\frac{\mathrm{A}}{2} \sin m\left(t-\frac{\mathrm{L}_{1}}{a_{1}}\right)-\frac{\mathrm{A}}{2} \sin m\left(t+\frac{\mathrm{L}_{1}}{a_{1}}\right)\right]$

$$
v_{1}=v_{10}+\frac{a}{a_{1}} \mathrm{~A} \sin m \frac{L_{1}}{a_{1}} \cos m t
$$

De même, en tenant compte de (8) :

(11a) $\quad v_{2}=v_{20}+\frac{! f}{u_{2}} \mathrm{~B} \cos m \frac{l_{2}}{a_{2}} \cos m t$.

Introduisons $v_{2}$ et $v_{2}$ dans l'équation de continuité (6); on trouve:

$\frac{\mathrm{S}_{\mathrm{1}}}{a_{1}} \mathrm{~A} \sin m \frac{\mathrm{I}_{1}}{a_{1}} \cos m t=\frac{\mathrm{S}_{2}}{a_{2}} \mathrm{~B} \cos m \frac{\mathrm{I}_{2}}{a_{2}} \cos m t$

Divisons par $\mathrm{A} \cos m \frac{\mathrm{L}_{1}}{a_{1}}=\mathrm{B} \sin m \frac{\mathrm{L}_{2}}{a_{2}}$ il vient :

$$
\frac{S_{1}}{a_{1}} \lg m \frac{L_{1}}{a_{1}}=\frac{S_{2}}{a_{2}} \operatorname{cotg} m \frac{L_{2}}{a_{2}}
$$

Posons : $\frac{S_{1}}{a_{1}} \frac{a_{2}}{s_{2}}=\frac{1}{\mu^{\prime}}$; on trouve :

(12a)

$$
\operatorname{tg} m \frac{\mathrm{l}_{L_{1}}}{a_{1}}=\mu^{\prime} \operatorname{cotg} m \frac{\mathrm{L}_{2}}{a_{2}}
$$

équation dont on peut tirer $m$, puis $\Theta$, période apparente du mouvement périodique.

On vérifiera que cette relation ne dépend que de la forme de la conduite, de ses sections, longueurs et de son élasticité.

Il est utile de comparer cette formule (12 a) avec la formule (11) de Foch, page 64 de son étude.

\section{2) Cas particuliers}

\section{a) $\mathrm{L}_{1}=o$ (conduite à caractéristique unique)}

On a $: \operatorname{cotg} m \frac{\mathrm{L}_{2}}{a_{2}}=o$; d'où : et :

$$
\begin{gathered}
m \frac{L_{2}}{a_{2}}=\frac{\pi}{2} \\
\Theta=\frac{2 \pi}{m}=4 \frac{L_{2}}{a_{2}}
\end{gathered}
$$

La période apparente du fondamental, dans une conduite de section constante, est égale à la période théorique $4 \frac{L}{a}$ de celle conduite.

b) Cas ou $\frac{L_{2}}{a_{1}}=\frac{L_{2}}{a_{s}}=\frac{\mathrm{L}}{2 a_{\mathrm{m}}}$

Nous désignons par $L=L_{1}+L_{2}$ la longueur totale et par a un une célérité moyenne satisfaisant à la relation posée plus haut. La formule (12a) devient :

$$
\text { tg } \frac{m \mathrm{~L}_{1}}{a_{3}}=\mu^{\prime} \operatorname{cotg} \frac{m \mathrm{~L}_{1}}{a_{2}} \text {; d'où : }
$$

$$
\begin{array}{r}
\operatorname{cotg} m \frac{L_{1}}{a_{1}}=\sqrt{\frac{T}{\mu^{\prime}}}=\frac{D_{1}}{D_{2}} \sqrt{\frac{a_{2}}{a_{1}}} \text { et : } \\
\Theta-\frac{2 \pi \frac{1}{a}}{\operatorname{arccatg} \frac{r}{D_{2}} \sqrt{\frac{r_{1}}{r_{2}}}}
\end{array}
$$

\begin{tabular}{|c|c|c|c|c|c|}
\hline Pour $\frac{D_{1}}{D_{2}}=1,2$ & 1,104 & 1 & 0,7 & 0,5 & 0,3 \\
\hline$\frac{1}{\mu^{\prime}}=1,18$ & 1,0 & 0,82 & 0,491 & 0,205 & $0,07 \dot{4}$ \\
\hline$\sqrt{\frac{1}{\mu^{\prime}}}=1,086$ & 1,0 & 0,905 & 0,635 & 0,4525 & 0,2718 \\
\hline$m \frac{L_{1}}{a_{1}}=\beta=47^{g} 38^{\prime}$ & $50^{g}$ & $53^{g} 1^{\prime}$ & $\left(i 4^{\prime} g\right.$ & $73^{g}$ & $83^{g}$ \\
\hline $0: \frac{4 \mathrm{~L}}{a_{m}}=1,056$ & 1 & 0,94 & 0,782 & 0,685 & 0,602 \\
\hline
\end{tabular}

Rappelons ici une formule que nous avons démontrée ailleurs $(16)$ :

$$
\sigma=p \frac{\mathrm{E}}{2}\left(\frac{g}{\gamma^{\mathrm{a}}}-\frac{1}{\varepsilon}\right)
$$

formule dans laquelle $\sigma$ est la tension dans l'acier de la conduite, et qui nous permel de calculer le rapport $\frac{a_{2}}{a_{1}}$ en fonction de la pression d'eau au point de bifurcation $\mathrm{A}$ et à l'oblurateur $O$ et des constantes connues $E, g$ et $\varepsilon$.

Pour fixer les idées, nous admettons, en utilisant la formule (14): $a_{1}=1000 \mathrm{~m} / \mathrm{sec}$. et $a_{2}=820 \mathrm{~m} / \mathrm{sec}$., ce qui est une solution admissible pour le cas $\frac{\mathrm{L}_{1}}{a_{1}}=\frac{\mathrm{L}_{2}}{a_{2}}$

On a : $\quad 1 / \mu^{\prime}=\frac{D^{2}{ }_{1}}{D^{2}{ }_{2}} \times \frac{820}{1000}=0.82 \frac{D^{2}{ }_{1}}{D^{2}{ }_{2}}$

Posons : $m \frac{L_{1}}{a_{l}}=\beta$, d'où $\Theta: \frac{4 \mathrm{~L}}{\mathrm{~m}}=\frac{2 \pi}{m} \times \frac{a_{\mathrm{m}}}{4 \mathrm{~L}}=\frac{\pi}{4 \beta}$ Nous pouvons dresser le tableau suivant:

(16) Charles Jazgen. - "La Houille Blanche " mars-avril 1934, formule (16), p. 37 . 


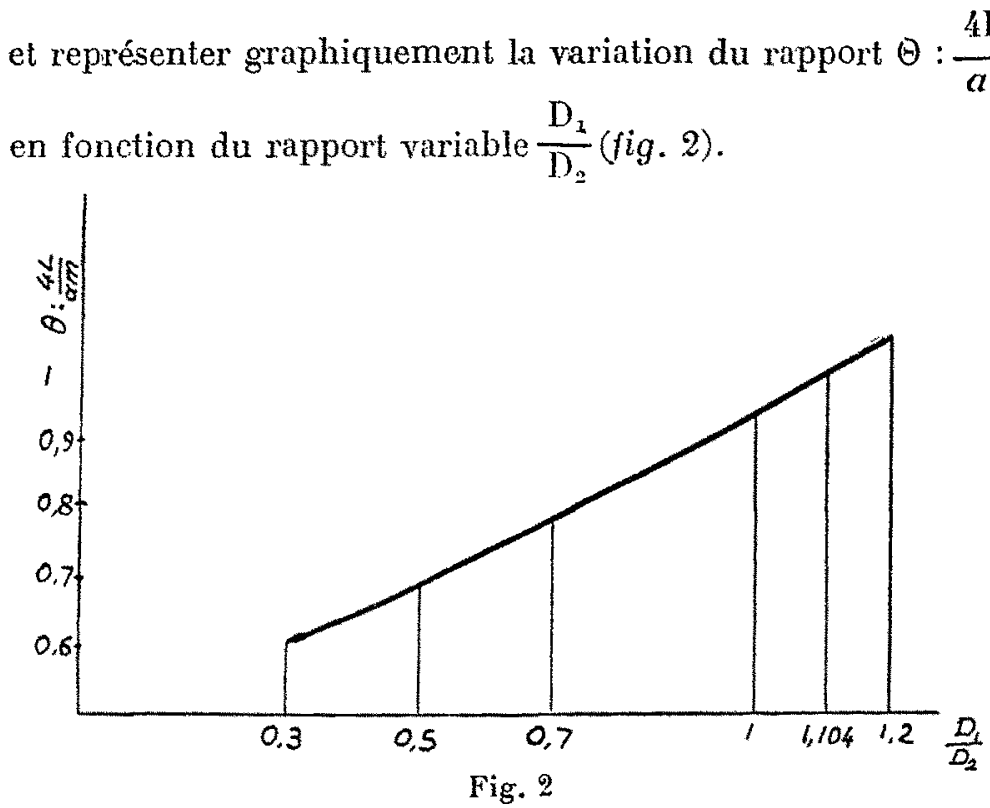

3) Cas général . exemples :

Nous avons trouvé, dans l'ouvrage de Camichel-EydouxGariel [11], mention de quelques rares mesures de périodes apparentes, qui nous permettent de contrôler notre formule (12a).

a) Conduite du Laboratoire de Toulouse (17):

La conduite de Toulouse se composail de deux sections " 1 " et " 2 ", dont voici les diamètres et longueurs :

$\mathrm{D}_{1}=0,40 \mathrm{~m} \cdot \mathrm{L}_{1}=105,85 \mathrm{~m}$;

$a_{1}=1,350 ; \frac{\mathrm{L}_{1}}{a_{1}}=0^{\prime \prime}, 078$

$\mathrm{D}_{2}=0,80 \mathrm{~m} . ; \mathrm{L}_{2}=201,6 \mathrm{~m}$;

$a_{2}=1,300 ; \frac{L_{2}}{a_{2}}=0^{\prime \prime}, 155$

On en tire :

$$
\frac{1}{\mu^{\prime}}=\frac{\Delta_{1} a_{2}}{S_{2} a_{1}}=\frac{\frac{0,4}{2} \times 1,300}{0,8 \times 1,356}=0,24
$$

Soit $\Theta=\frac{2 \pi}{m}$ la période apparente. Posons $\Theta=0^{\prime \prime} 71$; on a alors :

$$
\begin{gathered}
\frac{2 \pi}{\Theta} \frac{L_{1}}{a_{1}}=\frac{2 \pi \times 0,078}{0,71}=2 \pi \times 0,1099=44^{g} \text { et } \operatorname{tg} m \frac{L_{1}}{a_{1}}=0,827 \\
\frac{2 \pi}{\Theta} \frac{L_{2}}{a_{2}}=\frac{2 \pi \times 0,155}{0,71}=2 \pi \times 0,218=87^{g} 30 \text { et } \\
\operatorname{cotg} m \frac{L_{2}}{a_{2}}=0,2025
\end{gathered}
$$

valeurs qui vérifient la formule $\left(12_{2}\right)$.

Camichel avait mesuré $\Theta=0^{\prime \prime}, 69$ pour la période apparenle de la conduite. La période théorique était estimée à $\Theta=0^{\prime \prime}, 92$ :

\section{b) Conduite de Fully (18) :}

On peut définir la conduite de Fully, comme suit : $\mathrm{D}_{1}=0,50 \mathrm{~m} . ; \quad \mathrm{L}_{1}=2.347,5 \mathrm{~m}$;

$a_{1}=1,255 ; \frac{L_{1}}{a_{2}}=1^{\prime \prime}, 872$

(17) Cammomel-Expoux-Gamrez, p. 210.

(18) Canicule-Eyboux-Gamme, p. 219 et 376.
$D_{2}=0,60 \mathrm{~m} ; \mathrm{L}_{\mathrm{g}}=2,278,0 \mathrm{~m}$;

$a_{2}=1,0 ; 4 ; \frac{L_{2}}{a_{2}}=2^{\prime \prime}, 124$

$$
\frac{1}{\mu^{\prime}}=\frac{\frac{2}{0,5} \times 1,074}{\frac{2}{0,6^{2}} \times 1,255}=0,594
$$

Posons $\Theta=13^{1 / 75}$, on a, alors :

$$
\begin{gathered}
\frac{2 \pi}{\Theta} \frac{L_{1}}{a_{1}}=2 \pi \times 0,136=54^{g} 50 ; \operatorname{tg} m \frac{L_{1}}{a_{1}}=1,153 \\
\frac{2 \pi}{\Theta} \frac{L_{2}}{a_{2}}=2 \pi \times 0,1545=61^{g} 80 ; \operatorname{colg} m \frac{L_{2}}{a_{2}}=0,698
\end{gathered}
$$

L'équation $(12 a)$ se trouve satisfaite pour $\Theta=13^{\prime \prime}, 75$.

Boucher avait mesuré : $\Theta=13^{\prime \prime}, 5$.

La période théorique était : $\Theta=15^{\prime \prime}, 95$.

La concordance entre notre formule el les mesures effectuées il y a une vingtaine d'années nous paraît entièrement satisfaisante. Il en est de même pour les usines de Pau, Cauterets et Eget (19).

Pour la conduite d'Akersand, qui présente plusieurs disconlinuités, la concordance est un peu moins bonnc. Nous avons trouvé $\Theta=4^{\prime \prime}, 4$, alors que Neeser mesurait $\Theta=4^{\prime \prime}, 63$.

4) Cas d'une bifurcation.

Le cas de bifurcation se traite de façon analogue à celui d'une variation de section. Considérons les conduites 1 ,

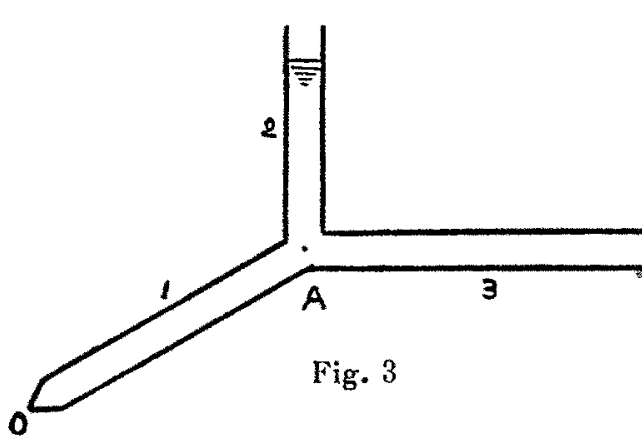
2 el 3 , bifurquant en $A$; l'obturateur se trouvant en 0 , à la base de 1 . Désignons, comme pré. cédemment, par $F(t)$ et $f(t)$ et $\operatorname{par} G(t)$ et $g(t)$ les fonctions d'intégration de l'équation fondamentale pour les conduiles 1 et 2 . Désignons par $H(t)$ ef $h(t)$ ces mêmes fonctions pour la conduite 3 . On a, alors, non seulement :

$$
\mathrm{F}\left(t-\frac{\mathrm{L}_{1}}{a_{1}}\right)+\mathrm{F}\left(t+\frac{\mathrm{L}_{1}}{a_{1}}\right)=\mathrm{G}(t)-\mathrm{G}\left(t-\frac{2 \mathrm{~L}_{2}}{a_{2}}\right)
$$

mais aussi :

d'où :

$$
=\mathrm{H}(t)-h\left(t-\frac{2 \mathrm{~L}_{3}}{a_{3}}\right)
$$

$\mathrm{A} \cos m \frac{\mathrm{I}_{1}}{a_{1}} \sin m t=\mathrm{B} \sin m \frac{\mathrm{L}_{2}}{a_{2}} \cos \left(m t+\varphi_{2}-m \frac{\mathrm{L}_{2}}{a_{2}}\right)$.

d'où l'on tire :

$$
=\mathrm{C} \sin m \frac{\mathrm{L}_{3}}{a_{3}} \cos \left(m t+\varphi_{\mathrm{s}}-m \frac{\mathrm{L}_{3}}{a_{3}}\right)
$$

$\mathrm{A} \cos m \frac{\mathrm{L}_{1}}{a_{1}}=\mathrm{B} \sin m \frac{\mathrm{L}_{2}}{a_{2}}=\mathrm{C} \sin m \frac{\mathrm{L}_{3}}{a_{3}}$

On a, d'autre part :

$\mathrm{S}_{1} \sqrt{1}_{1}=\mathrm{S}_{1} V_{1},+\mathrm{S}_{1} \quad \frac{g}{a_{1}} \mathrm{~A} \sin m \frac{\mathrm{L}_{1}}{a_{1}} \cos m t$

(19) Camicher, Exdoux, Gantet, p. 241-242. 
$\mathrm{S}_{2} \sqrt[V]{2}_{2}=\mathrm{S}_{2} V_{2}+\mathrm{S}_{2} \frac{g}{n_{2}} \mathrm{~B} \cos m \frac{\mathrm{I}_{2}}{a_{2}} \cos m l$

$\mathrm{S}_{3} \sqrt{3}_{3}=\mathrm{S}_{3} V_{3}+\mathrm{S}_{3} \frac{a}{a_{3}} \mathrm{C} \cos m \frac{\mathrm{L}_{33}}{a_{3}} \cos m l ;$ d'ou :

$\frac{\mathrm{S}_{\mathrm{L}}}{a_{1}} \mathrm{~A} \sin m \frac{\mathrm{L}_{1}}{a_{1}}=\frac{\mathrm{S}_{2}}{a_{2}} \mathrm{~B} \cos m \frac{\mathrm{L}_{2}}{a_{2}}+\frac{\mathrm{S}_{3}}{a_{3}} \mathrm{C} \cos m \frac{\mathrm{L}_{3}}{a_{3}}$

Divisons par : $\mathrm{A} \cos m \frac{\mathrm{l}_{1}}{a_{1}}=\mathrm{B} \sin m \frac{\mathrm{I}}{a_{2}}=\mathrm{G} \sin m \frac{\mathrm{L}_{3}}{a_{3}}$ on trouve :

$$
\frac{\mathrm{S}_{1}}{a_{1}} \lg m \frac{\mathrm{L}_{1}}{a_{1}}=\frac{\mathrm{S}_{2}}{a_{2}} \operatorname{cotg} m \frac{\mathrm{L}_{2}}{a_{2}}+\frac{\mathrm{S}_{3}}{a_{3}} \operatorname{cotg} m \frac{\mathrm{L}_{3}}{a_{3}}
$$

ou, en posant : $\frac{S_{2}}{a_{2}} \frac{a_{3}}{S_{1}}=\mu^{\prime}$ et $\frac{S_{3}}{a_{3}} \frac{a_{1}}{S_{1}}=\mu^{\prime \prime}$ $\operatorname{tg} m \frac{\mathrm{L}_{1}}{a_{1}}=\mu^{\prime} \operatorname{cotg} m \frac{\mathrm{L}_{2}}{a_{2}}+\mu^{\prime \prime} \operatorname{cotg} m \frac{\mathrm{L}_{3}}{a_{3}}$

(A suivre)

\title{
ÉLECTRICITÉ
}

Notes de Métrologie Industrielle

\section{Le contrôle économique de la Production dans les Usines interconnectées}

\section{MÉTHODES ET DISPOSITIFS}

\author{
par M. Dugrt, Ingénieur I. E. G.
}

\section{Generalites}

Suivant 'leurs disponibilités, les Usines interconnectées deivent fournir au Réseau qu'elles alimentent des contingents d'énergie journaliers, généralement différents, d'après des conventions et des consignes préalablement concertées.

Le service de la Répartition du Réseau désigne les Usines qui doivent concourir à ta livraison du courant et fixe l'importance de leurs interventions respectives. Il appartient aux Chefs d'Exploitation des Stations Génératrices de meltre judicieusement en service et en charge les groupes générateurs pour assurer, dans les conditions les plus économiques, la fourniture requise.

Sous un certain aspect, que cette note précisera, et d'un point de vuc essentiellement pratique, l'une quelconque des stations génératrices est, au Réseau qu'elle alimente, ce qu'un générateur quelconque est, individuellement, à l'Usine même sur les barres de laquelle il débite.

Or, pour assurer sur un réseau de distribution, la fourniture d'une puissance déterminée, il est une infinité de manières de répartir la charge entre les groupes générateur des Usines interconnectées. A chaque répartition correspond une valeur bien déterminée du rendement du système. Mais il est une répartition et une seule pour laquelle ce rendement est maximum : répartition optima de la puissance totale entre lies Usines interconnectées, répartition optima de la charge de chaque Usine entre les divers groupes générateurs ıqui l'équipent.

La détermination et le contrôle des conditions de répartition optima peurvent être pratiquement réalisés par une méthodes et desl dispositifs simples. Exposer le principe de l'une et des autres, tel esi le but de la communication que voici.
L'intérêt de la question est absolument général. Vainement, objecterait-on le cas d'unités identiques de mêmes caractéristiques, de même provenance etc... Considérations purement idéales, car pratiquement, les groupes et les Usines interconnectées, accuseront toujours des différences sensibles. L'expérience révèlera, généralement, dans leurs conditions de fonctionnement respectives, des particularités suffisantes pour justifier un contrôle systématique de la répartition des charges et du rendement

\section{Le Problème Général}

\section{de la Répartition des Charges et du Rendement} dans les

Systèmes générateurs complexes couplés en parallèle

Considérons $\mathrm{R}$ ensembles de générateurs :

$\mathrm{G}^{1}{ }_{1}, \mathrm{G}^{1}{ }_{2}, \mathrm{G}^{1}, \ldots \ldots \ldots \ldots \ldots, \mathrm{G}^{\mathrm{1}}{ }_{\mathrm{k}} \ldots \ldots \ldots \ldots \mathrm{G}_{\mathrm{k} 1}^{\mathrm{x}}$

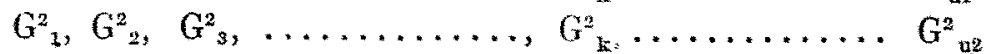

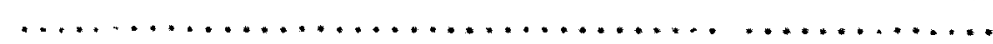

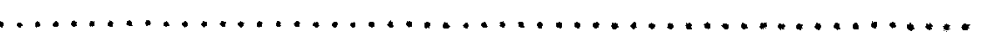
$G^{n}, G^{n}{ }^{n}, G^{n}, \ldots \ldots \ldots \ldots, G^{n}{ }_{k} \ldots \ldots \ldots \ldots, G^{n}{ }_{n n}$ $\mathrm{G}^{\mathrm{r}}{ }_{\mathrm{l}}, \mathrm{G}^{\mathrm{r}}, \quad \mathrm{G}^{\mathrm{r}}, \ldots \ldots \ldots \ldots, \mathrm{G}_{\mathrm{k}}^{\mathrm{r}} \ldots \ldots \ldots \ldots \mathrm{G}^{\mathrm{r}}{ }_{\mathrm{urr}}$ couplés respectivement sur les barres omnibus ide $r$ stations génératrices.

$\mathrm{S}_{1}, \mathrm{~S}_{2}, \mathrm{~S}_{3}, \ldots \ldots \ldots \ldots \mathrm{S}_{\mathrm{n}} \ldots \ldots \ldots \ldots \mathrm{S}_{\mathrm{r}}$ interconnectées pour l'alimentation d'un réseau de distribution d'énergie électrique. 